QUARTERLY OF APPLIED MATHEMATICS

VOLUME LXIII, NUMBER 2

JUNE 2005, PAGES 325-333

S 0033-569X(05)00954-1

Article electronically published on February 18, 2005

\title{
REMARKS ON INSTABILITIES AND ELLIPTICITY FOR A FIBER-REINFORCED COMPRESSIBLE NONLINEARLY ELASTIC SOLID UNDER PLANE DEFORMATION
}

\author{
BY \\ J. MERODIO (Department of Structural and Mechanical Engineering, E.T.S. de Ingenieros \\ Industriales y de Telecomunicación, University of Cantabria, 39005, Santander, Spain) \\ AND \\ R. W. OGDEN (Department of Mathematics, University of Glasgow, Glasgow G12 8QW, United \\ Kingdom)
}

\begin{abstract}
In this paper we examine loss of ellipticity and associated failure for fiber-reinforced compressible nonlinearly elastic solids under plane deformation. The analysis concerns a material model that consists of an isotropic base material augmented by a reinforcement dependent on the fiber direction. We examine reinforcement that introduces additional stiffness under shear deformations. It is shown that loss of ellipticity may be associated with, in particular, a weak surface of discontinuity normal to or parallel to the deformed fiber direction or at an intermediate angle. More particularly, under uniaxial loading in the fiber direction loss of ellipticity may be associated with different failure mechanisms. Under compression these include fiber kinking and fiber splitting, while under extension the relevant mechanism is matrix failure.
\end{abstract}

1. Introduction. This paper completes a series of articles [1] -6$]$ that deal with fiber instabilities in fiber-reinforced nonlinearly elastic solids under plane deformation. In particular, a continuum mechanical model within the framework of nonlinear elasticity has been proposed to capture fiber failure in these materials. Fiber failure is assumed to occur at loss of ellipticity of the governing differential equations. At the breakdown of ellipticity, surfaces of discontinuity arise inside the material. Depending on the loading regime and on the direction of the normal to these surfaces relative to the fiber direction, the mechanism of failure is interpreted variously in terms of fiber kinking, fiber splitting, fiber de-bonding or matrix failure. In the past few years there has been considerable interest in modelling fiber instability or fiber failure in fiber-reinforced materials, but, to

Received June 18, 2004.

2000 Mathematics Subject Classification. Primary 74B20.

Key words and phrases. Nonlinear elasticity, loss of ellipticity, fiber reinforcement, reinforcing models, transverse isotropy.

E-mail address: merodioj@unican.es

E-mail address: rwo@maths.gla.ac.uk

(C)2005 Brown University 
our knowledge, the above-cited papers provide the first treatment that enables all these mechanisms to be captured within a single theory. Detailed references to the literature are given in these papers. We mention here, however, that recently an attempt has been made to classify composite compressive failure modes as a basis for predicting such failure [7]. The approach is based on linear elasticity and it concludes that the type of failure (kinking or splitting) depends on, amongst other factors (such as geometrical quantities), the shear response of the matrix and hence the shear modulus of the composite.

In three dimensions, two independent deformation invariants are required to characterize the anisotropic nature of a transversely isotropic material. We denote these by $I_{4}$ and $I_{5}$, which are taken together with the three invariants $I_{1}, I_{2}, I_{3}$ of the Cauchy-Green deformation tensors associated with isotropy in a compressible material. For plane deformations only two of $I_{1}, I_{2}, I_{3}$ are independent and, if the fiber direction is taken to be in the considered plane, $I_{4}$ and $I_{5}$ are connected through $I_{1}$ and $I_{3}$ [2]. The in-plane part of the material response may then be regarded as depending only on $I_{1}, I_{2}$ and $I_{4}$ or on any equivalent set of three independent invariants. The ellipticity of the governing twodimensional equations in this case then depends on only one anisotropic invariant (either $I_{4}$ or $I_{5}$ ). In our analysis a constitutive model consisting of an isotropic base material augmented by a uniaxial reinforcement depending on only one of the two anisotropic invariants has been used.

The analysis for incompressible materials was contained in [1], where, for anisotropy characterized by $I_{4}$, under uniaxial loading in the fiber direction, it was shown that (a) the failure mechanism captured in compression is fiber kinking and (b) in tension it is fiber de-bonding. On the other hand, for anisotropy characterized by $I_{5}$ under the same loading, we found that (a) compressive failure is associated with fiber kinking and fiber splitting and (b) tensile failure is associated with shear bands in the sense that the discontinuity surfaces are not close to either the fiber direction or its normal. In [2], a similar analysis was carried out for compressible materials. In this case, for $I_{4}$-based anisotropy, we found the same results as for incompressible materials. A simplified form of $I_{5}$-based anisotropy was chosen, and, as for incompressible materials, it was shown that compressive failure may be associated with fiber kinking and fiber splitting. This modified form of $I_{5}$ couples the invariants $I_{1}$ and $I_{4}$. In the present paper we provide an analysis for the anisotropy based on $I_{5}$ itself, which combines the invariants $I_{1}, I_{3}$ and $I_{4}$. The inclusion of the invariant $I_{3}$ in this case modifies the analysis both qualitatively and quantitatively.

In Section 2, the material model is introduced and the ellipticity, strong ellipticity and loss of ellipticity conditions for the governing differential equations are summarized, including their specializations to plane strain. In Section 3, the ellipticity status of a general reinforcing model depending on $I_{5}$ is established. As distinct from the results obtained for the simplified form of $I_{5}$-based anisotropy, we find here that loss of ellipticity is possible for convex reinforcing models when $I_{5}>1$. However, this is not so in the special case of uniaxial tensile loading in the fiber direction, for which loss of ellipticity does require loss of convexity of the reinforcing model. Furthermore, loss of ellipticity is associated with a discontinuous surface normal to the fiber direction and it is interpreted 
as matrix failure. Under fiber contraction it is found that failure of ellipticity may occur in two different modes, which may be associated with fiber kinking and fiber splitting.

In Section 4 we summarize and discuss briefly the results obtained in the previous sections.

2. Constitutive equations and ellipticity. For a compressible elastic solid we consider, in the reference configuration, a single fiber direction characterized by a unit vector field, which we denote by $\mathbf{A}$. The strain-energy function (defined per unit reference volume) is denoted by $W$ and depends on the five independent invariants mentioned in Section 1 , denoted $I_{1}, I_{2}, I_{3}, I_{4}, I_{5}$, and we write

$$
W=W\left(I_{1}, I_{2}, I_{3}, I_{4}, I_{5}\right) .
$$

We emphasize that $I_{1}, I_{2}$ and $I_{3}$ are the principal invariants of the left Cauchy-Green deformation tensor $\mathbf{B}=\mathbf{F F}^{T}$ (or of the right Cauchy-Green deformation tensor $\mathbf{C}=$ $\mathbf{F}^{T} \mathbf{F}$ ), where $\mathbf{F}$ is the deformation gradient tensor relative to the natural (unstressed) configuration, which we adopt as the reference configuration. Thus,

$$
I_{1}=\operatorname{tr} \mathbf{C}, \quad I_{2}=\frac{1}{2}\left[(\operatorname{tr} \mathbf{C})^{2}-\operatorname{tr}\left(\mathbf{C}^{2}\right)\right], \quad I_{3}=\operatorname{det} \mathbf{C} .
$$

The invariants $I_{4}$ and $I_{5}$ are associated with the fiber reinforcement and depend on $\mathbf{A}$ as well as on $\mathbf{C}$. They are defined by

$$
I_{4}=\mathbf{a} \cdot \mathbf{a}=\mathbf{A} \cdot(\mathbf{C A}), \quad I_{5}=\mathbf{a} \cdot(\mathbf{B a})=\mathbf{A} \cdot\left(\mathbf{C}^{2} \mathbf{A}\right),
$$

where the notation $\mathbf{a}$ is defined by $\mathbf{a}=\mathbf{F A}$.

We note that $\sqrt{I_{4}}$ has an immediate interpretation as the stretch in the direction $\mathbf{A}$, as can be seen from (3) ${ }_{1}$. Thus, $I_{4}$ registers deformations that modify the length of the fiber. In particular, in terms of rectangular Cartesian basis vectors $\mathbf{i}_{1}, \mathbf{i}_{2}, \mathbf{i}_{3}$, with $\mathbf{A}=\mathbf{i}_{1}$, we have simply $I_{4}=C_{11}$. Similarly, $I_{5}=C_{11}^{2}+C_{12}^{2}+C_{13}^{2}$. Hence, in general, $I_{5}$ registers changes in the fiber reinforcement length by means of the indicator $C_{11}$ and shear deformations via the indicators $C_{12}$ and $C_{13}$.

2.1. Equilibrium and ellipticity. In the absence of body forces the equation of equilibrium may be expressed in the form $\operatorname{Div} \mathbf{S}=\mathbf{0}$, where

$$
\mathbf{S}=\frac{\partial W}{\partial \mathbf{F}}
$$

is the nominal stress tensor. In components, the equilibrium equation may be written

$$
\mathcal{A}_{\alpha i \beta j} x_{j, \alpha \beta}=0
$$

where

$$
\mathcal{A}_{\alpha i \beta j}=\frac{\partial^{2} W}{\partial F_{i \alpha} \partial F_{j \beta}}
$$

are the components of the tensor of elastic moduli associated with the nominal stress and deformation gradient. In the above component expressions, Greek and Roman indices are associated with the reference and deformed configurations, respectively. A subscript such as $\alpha$ following a comma indicates differentiation with respect to the coordinate $X_{\alpha}$, where $\left(X_{1}, X_{2}, X_{3}\right)$ are the components of the position vector $\mathbf{X}$ of a material particle in the reference configuration. The corresponding position vector in the deformed configuration 
is denoted $\mathbf{x}$ and has components $\left(x_{1}, x_{2}, x_{3}\right)$. The usual summation convention for repeated indices is also adopted.

Let the reference configuration of a material body be denoted by $\mathcal{B}_{r}$. Suppose that $\mathbf{F}$ is continuous in $\mathcal{B}_{r}$ and that $\operatorname{Grad} \mathbf{F}$, with components $x_{i, \alpha \beta}$, is also continuous except possibly across a surface $\mathcal{S}_{r}$ in $\mathcal{B}_{r}$ where it may suffer a discontinuity in one or more of its components. The surface in question is referred to as a weak discontinuity. Let the normal vector to $\mathcal{S}_{r}$ be denoted by $\mathbf{N}$. Then, the jump in $x_{i, \alpha \beta}$ across $\mathcal{S}_{r}$ has the specific form

$$
\left[x_{i, \alpha \beta}\right]=m_{i} N_{\alpha} N_{\beta},
$$

where $[\cdot]$ denotes the difference in the enclosed quantity evaluated on the two sides of $\mathcal{S}_{r}$ and $m_{i}$ are the components of a vector $\mathbf{m}$ that characterizes the discontinuity.

On applying (7) to the left-hand side of (5), we obtain

$$
\mathcal{A}_{\alpha i \beta j} m_{i} N_{\alpha} N_{\beta}=0 \text {. }
$$

We now make use of the push forward of $\mathcal{A}_{\alpha i \beta j}$ to the deformed configuration, denoted $\mathcal{A}_{0 p i q j}$ and defined by

$$
J \mathcal{A}_{0 p i q j}=F_{p \alpha} F_{q \beta} \mathcal{A}_{\alpha i \beta j}
$$

(see, for example, 8]). If now we define the vector $\mathbf{n}$ by

$$
\mathbf{N}=\mathbf{F}^{T} \mathbf{n},
$$

then, on use of Nanson's formula $\mathbf{n d} a=J \mathbf{F}^{-T} \mathbf{N} \mathrm{d} A$ for the deformation of area elements, we identify $\mathbf{n}$ as the corresponding normal vector to the image of $\mathcal{S}_{r}$ in the deformed configuration. We emphasize that in Nanson's formula $\mathbf{N}$ and $\mathbf{n}$ are unit vectors, but that the $\mathbf{n}$ defined by (10) is not in general a unit vector.

On use of equations (9) and (10) we may write (8) in the compact form

$$
\mathbf{Q}(\mathbf{n}) \mathbf{m}=\mathbf{0},
$$

wherein we have introduced the acoustic tensor, denoted $\mathbf{Q}(\mathbf{n})$, which has components given by

$$
Q_{i j}=\mathcal{A}_{0 p i q j} n_{p} n_{q}
$$

It follows from (11) that the existence of a surface of weak discontinuity requires that $\mathbf{Q}(\mathbf{n})$ be singular for some non-zero $\mathbf{n}$. The associated values of $\mathbf{n}$ are given by

$$
\operatorname{det} \mathbf{Q}(\mathbf{n})=0 .
$$

For each $\mathbf{n}$ determined from (13) the corresponding $\mathbf{m}$ is found from (11). Thus, for a deformation with the considered properties to be admissible, the equality

$$
\mathcal{A}_{0 \text { piqj }} n_{p} n_{q} m_{i} m_{j} \equiv[\mathbf{Q}(\mathbf{n}) \mathbf{m}] \cdot \mathbf{m}=0
$$

must therefore hold for some pair of non-zero vectors $\mathbf{m}$ and $\mathbf{n}$. This equation, together with (11), defines pairs of non-zero vectors $\mathbf{m}$ and $\mathbf{n}$, and we point out that $\mathbf{m}$ is a null eigenvector of $\mathbf{Q}(\mathbf{n})$ for $\mathbf{n}$ satisfying (13).

No such solutions exist when the system of equations (5) is elliptic, i.e. if the condition

$$
[\mathbf{Q}(\mathbf{n}) \mathbf{m}] \cdot \mathbf{m} \equiv \mathcal{A}_{0 p i q j} n_{p} n_{q} m_{i} m_{j} \neq 0
$$


holds for all vectors $\mathbf{m} \neq \mathbf{0}, \mathbf{n} \neq \mathbf{0}$. The strong-ellipticity condition is the stronger requirement

$$
[\mathbf{Q}(\mathbf{n}) \mathbf{m}] \cdot \mathbf{m} \equiv \mathcal{A}_{0 p i q j} n_{p} n_{q} m_{i} m_{j}>0, \quad \mathbf{m} \neq 0, \mathbf{n} \neq 0 .
$$

Thus, if the governing equations are (equivalently, the strain-energy function is) strongly elliptic, then $\mathbf{Q}(\mathbf{n})$ is positive definite for all vectors $\mathbf{n} \neq \mathbf{0}$. Without loss of generality we take $\mathbf{m}$ and $\mathbf{n}$ to be unit vectors.

To summarize, we remark that analysis of equation (15) for particular forms of strainenergy function $W$ determines the ellipticity status of that particular energy function. A deformation gradient $\mathbf{F}$ satisfying (15) for every pair of unit vectors $\mathbf{m}$ and $\mathbf{n}$ is said to be an elliptic deformation for that $W$. If all possible deformations for some $W$ are elliptic, then the material itself is referred as an elliptic material. Moreover, the vector $\mathbf{n}$ is identified as a normal vector to a weak surface (in the deformed configuration) associated with the discontinuity (7). We emphasize that if $\mathbf{n}$ is a unit vector, then $\mathbf{N}$, defined by (10), is not in general a unit vector.

Note that a surface of strong discontinuity, that is, a surface across which $\mathbf{F}$ itself is discontinuous, is also defined by the loss of ellipticity condition (14). Indeed, a discontinuity in $\mathbf{F}$ implies that its derivative is also discontinuous, and hence a surface of strong discontinuity (or strong surface) is also a weak surface.

2.1.1. Plane strain. We now specialize the above considerations to the case of plane strain deformations and, in particular, we examine the ellipticity of the considered material model for the situation in which the fiber direction is in the considered plane. Suppose that the plane in question corresponds to the $\left(X_{1}, X_{2}\right)$ coordinate plane so that the deformation is such that $x_{3}=X_{3}$, with $\left(x_{1}, x_{2}\right)$ independent of $X_{3}$. Then, the out-of-plane components of $\mathbf{F}$ are $F_{13}=F_{23}=F_{31}=F_{32}=0$ and $F_{33}=1$, and the corresponding components of $\mathbf{C}$ are $C_{13}=C_{23}=0$ and $C_{33}=1$. The out-of-plane principal stretch is $\lambda_{3}=1$. It then follows that

$$
I_{2}=I_{1}+I_{3}-1, \quad I_{5}=\left(I_{1}-1\right) I_{4}-I_{3},
$$

the first connection being well known, while the latter was given in [2].

Because of the connections (17), the strain energy $W\left(I_{1}, I_{2}, I_{3}, I_{4}, I_{5}\right)$ of an elastic material reinforced by a single family of fibers (in other words, a transversely isotropic elastic material) can be represented in terms of three independent invariants in the plane strain specialization. We may therefore write

$$
\hat{W}\left(I_{1}, I_{3}, I_{4}\right)=W\left(I_{1}, I_{1}+I_{3}-1, I_{3}, I_{4},\left(I_{1}-1\right) I_{4}-I_{3}\right),
$$

wherein the notation $\hat{W}$ is defined.

In terms of $\hat{W}$, the in-plane components of $\mathcal{A}_{0 p i q j}$ given by (9) with (6) are explicitly

$$
\begin{aligned}
J \mathcal{A}_{0 p i q j}= & 4 \hat{W}_{11} B_{p i} B_{q j}+4 I_{3} \hat{W}_{13}\left(B_{p i} \delta_{q j}+B_{q j} \delta_{p i}\right) \\
& +4 I_{3}^{2} \hat{W}_{33} \delta_{p i} \delta_{q j}+4 I_{3} \hat{W}_{34}\left(\delta_{p i} a_{q} a_{j}+\delta_{q j} a_{p} a_{i}\right) \\
& +4 \hat{W}_{14}\left(B_{p i} a_{q} a_{j}+B_{q j} a_{p} a_{i}\right)+4 \hat{W}_{44} a_{p} a_{i} a_{q} a_{j} \\
& +2 \hat{W}_{1} \delta_{i j} B_{p q}+2 I_{3} \hat{W}_{3}\left(2 \delta_{p i} \delta_{q j}-\delta_{p j} \delta_{q i}\right)+2 \hat{W}_{4} \delta_{i j} a_{p} a_{q},
\end{aligned}
$$

where $\delta_{i j}$ denotes the Kronecker delta. In (19) and henceforth indices take the values 1 and 2 only. 
Similarly, in terms of $\hat{W}$ and the components of $\mathbf{n}$ referred to the principal axes of $\mathbf{B}$, the components of $\mathbf{Q}$ given by (19) and (12) are

$$
\begin{aligned}
Q_{i j} & =4 \hat{W}_{11} \lambda_{i}^{2} \lambda_{j}^{2} n_{i} n_{j}+4 I_{3} \hat{W}_{13}\left(\lambda_{i}^{2}+\lambda_{j}^{2}\right) n_{i} n_{j} \\
& +4 I_{3}^{2} \hat{W}_{33} n_{i} n_{j}+4 I_{3} \hat{W}_{34}(\mathbf{n} \cdot \mathbf{a})\left(n_{i} a_{j}+n_{j} a_{i}\right) \\
& +4 \hat{W}_{14}(\mathbf{n} \cdot \mathbf{a})\left(\lambda_{i}^{2} n_{i} a_{j}+\lambda_{j}^{2} n_{j} a_{i}\right)+4 \hat{W}_{44}(\mathbf{n} \cdot \mathbf{a})^{2} a_{i} a_{j} \\
& +2 \hat{W}_{1} \delta_{i j}\left(\lambda_{1}^{2} n_{1}^{2}+\lambda_{2}^{2} n_{2}^{2}\right)+2 I_{3} \hat{W}_{3} n_{i} n_{j}+2 \hat{W}_{4} \delta_{i j}(\mathbf{n} \cdot \mathbf{a})^{2} .
\end{aligned}
$$

The strong ellipticity condition (16), specialized to the considered plane, then becomes

$$
\begin{aligned}
& 2 \hat{W}_{11}[\mathbf{m} \cdot(\mathbf{B n})]^{2}+4 I_{3} \hat{W}_{13}[\mathbf{m} \cdot(\mathbf{B n})](\mathbf{m} \cdot \mathbf{n})+2 I_{3}^{2} \hat{W}_{33}(\mathbf{m} \cdot \mathbf{n})^{2} \\
& +\left[4 I_{3} \hat{W}_{34}(\mathbf{m} \cdot \mathbf{n})+4 \hat{W}_{14}[\mathbf{m} \cdot(\mathbf{B n})]+2 \hat{W}_{44}(\mathbf{m} \cdot \mathbf{a})(\mathbf{n} \cdot \mathbf{a})\right](\mathbf{m} \cdot \mathbf{a})(\mathbf{n} \cdot \mathbf{a}) \\
& +\hat{W}_{1} \mathbf{n} \cdot(\mathbf{B n})+I_{3} \hat{W}_{3}(\mathbf{m} \cdot \mathbf{n})^{2}+\hat{W}_{4}(\mathbf{n} \cdot \mathbf{a})^{2}>0
\end{aligned}
$$

for all (in-plane) unit vectors $\mathbf{m}$ and $\mathbf{n}$, and we recall that $\mathbf{a}=\mathbf{F A}$, which is also an in-plane vector.

3. $I_{5}$-reinforcement. A possible reinforcing model for compressible materials, which is the counterpart of that used in the incompressible theory [1], is given by

$$
\hat{W}\left(I_{1}, I_{3}, I_{4}\right)=W_{\text {iso }}\left(I_{1}, I_{3}\right)+W_{\text {fib }}\left(I_{5}\right)
$$

where $I_{5}=\left(I_{1}-1\right) I_{4}-I_{3}$. Thus, there is a coupling of $\left(I_{1}, I_{3}\right)$ and $I_{4}$ through $I_{5}$. For convenience, we use the notation

$$
E\left(I_{1}, I_{3}\right)=W_{\text {iso }}\left(I_{1}, I_{3}\right), \quad G\left(I_{5}\right)=W_{\text {fib }}\left(I_{5}\right),
$$

as in 2], so that (22) is replaced by

$$
\hat{W}\left(I_{1}, I_{3}, I_{4}\right)=E\left(I_{1}, I_{3}\right)+G\left(I_{5}\right) .
$$

In [6] it was shown that the contribution of the term in $G$ to the normal Cauchy stress in the deformed fiber direction is $4 J^{-1} I_{5} G^{\prime}$. It is therefore appropriate to assume that

$$
G^{\prime}\left(I_{5}\right)>0(<0) \text { for } I_{5}>1(<1), \quad G^{\prime}(1)=0,
$$

and that

$$
G^{\prime}\left(I_{5}\right) \rightarrow-\infty(\infty) \text { as } \quad I_{5} \rightarrow 0(\infty) .
$$

Note, however, that $I_{5}>1$ does not in general correspond to fiber extension, although $I_{4}>1$ implies $I_{5}>1$ and $I_{5}<1$ implies $I_{4}<1$, as in the incompressible situation [1.

On substitution of (22) into (16) we obtain

$$
\left(\mathbf{Q}^{\mathrm{iso}} \mathbf{m}\right) \cdot \mathbf{m}+\left(\mathbf{Q}^{\mathrm{fib}} \mathbf{m}\right) \cdot \mathbf{m}>0,
$$

where $\mathbf{Q}^{\text {iso }}$ and $\mathbf{Q}^{\text {fib }}$ are now derived from $E$ and $G$, respectively.

We are particularly interested in strong reinforcement, in which case the reinforcing model provides the dominant contribution to the energy. It is therefore appropriate to neglect the influence of $E=W_{\text {iso }}$. This approximation enables considerable insight to be gained into failure mechanisms for strongly-reinforced solids. In particular, using (21) 
and omitting the contribution of $E$, we obtain the strong ellipticity condition for $G$ in the form

$$
\begin{aligned}
& 2 G^{\prime \prime}\left(I_{5}\right)\left[I_{4} \mathbf{m} \cdot(\mathbf{B n})+\left(I_{1}-1\right)(\mathbf{m} \cdot \mathbf{a})(\mathbf{n} \cdot \mathbf{a})-I_{3}(\mathbf{m} \cdot \mathbf{n})\right]^{2} \\
& +G^{\prime}\left(I_{5}\right)\left[\left(I_{1}-1\right)(\mathbf{n} \cdot \mathbf{a})^{2}+I_{4} \mathbf{n} \cdot(\mathbf{B n})\right. \\
& \left.+4 \mathbf{m} \cdot(\mathbf{B n})(\mathbf{m} \cdot \mathbf{a})(\mathbf{n} \cdot \mathbf{a})-I_{3}(\mathbf{m} \cdot \mathbf{n})^{2}\right]>0
\end{aligned}
$$

for all in-plane unit vectors $\mathbf{m}$ and $\mathbf{n}$ as the appropriate approximation of the strong ellipticity condition. It is clear that the coefficient of $G^{\prime}$ may be positive or negative regardless of the value of $I_{5}$, and that of $G^{\prime \prime}$ may be non-negative. This allows the possibility of failure of ellipticity in plane strain if $G$ is convex $\left(G^{\prime \prime}\left(I_{5}\right) \geq 0\right)$ and $I_{5}>1$, unlike the analogous situation for $F\left(I_{4}\right)$ [2]. However, as we show below, under uniaxial tensile loading in the fiber direction, loss of ellipticity does require that convexity of the reinforcing model $G\left(I_{5}\right)$ has been lost at a prior deformation. It remains an open question to determine (either for compressible or incompressible materials) whether a necessary condition for loss of ellipticity when $I_{5}>1$ and $I_{4}>1$ for general plane deformations is that $G$ is non-convex.

We first note the special case of (28) for which $\mathbf{n} \cdot \mathbf{a}=0$. In contrast to the corresponding situation for $F$, the terms in $G$ in (28) do not vanish and reduce to

$$
2 G^{\prime \prime}\left[I_{4} \mathbf{m} \cdot(\mathbf{B n})-I_{3}(\mathbf{m} \cdot \mathbf{n})\right]^{2}+G^{\prime}\left[I_{4} \mathbf{n} \cdot(\mathbf{B n})-I_{3}(\mathbf{m} \cdot \mathbf{n})^{2}\right] .
$$

In [2], it was noted that $F$ does not admit a weak surface aligned with the fiber direction. This is not the case for $G$. In particular, we focus first on fiber compression and recall that $G^{\prime}\left(I_{5}\right)<0$ for $I_{5}<1$. For this purpose we include the contribution of $E$. If, for example, we take $\mathbf{m}=\hat{\mathbf{a}}$, where $\hat{\mathbf{a}}=\mathbf{a} /|\mathbf{a}|$, and let $\mathbf{n}$ coincide with the principal axis of $\mathbf{B}$ corresponding to the stretch $\lambda_{1}$, then the left-hand side of (27), coupled with (29), simplifies to $\left(E_{1}+G^{\prime} I_{4}\right) \lambda_{1}^{2}$. If the isotropic base material is taken to be elliptic, then $E_{1}>0$, and since $G^{\prime}(1)=0$, this expression is positive in the undeformed configuration but can vanish as $I_{5}$ decreases from unity, at which point ellipticity is lost and the associated weak surface is aligned with the fiber direction. As in 1] we identify this failure of ellipticity with fiber splitting (Lee et al. [9]).

If we now consider $\mathbf{n}=\hat{\mathbf{a}}$ and interchange the roles of $\mathbf{m}$ and $\mathbf{n}$ in the above paragraph (with $\mathbf{n}$ still corresponding to stretch $\lambda_{1}$ ), the left-hand side of (27), combined with (29), becomes $E_{1} \lambda_{1}^{2}+G^{\prime} I_{4}\left(2 \lambda_{1}^{2}+\lambda_{2}^{2}\right)$. In this case ellipticity can fail as $I_{5}$ decreases at a value closer to unity than for the above example. This corresponds to a weak surface normal to the fiber direction. If the fiber is under contraction, this failure of ellipticity can correspond to fiber kinking, as for the $F\left(I_{4}\right)$ reinforcement.

The above analysis can easily be shown to agree with the loss of ellipticity analysis under uniaxial compressive loading in the fiber direction. It is in this sense that the breakdown of ellipticity is related to the failure mechanisms.

Fiber splitting and fiber kinking may occur simultaneously (Lee et al. 9]), in which case this may be predicted by having, simultaneously, weak surfaces both aligned with and normal to the fiber direction at the same deformation. Nevertheless, by the above analysis fiber kinking can in general be expected before fiber splitting. 
If the degree of anisotropy is sufficiently strong, then the terms in $G$ dominate the left-hand side of (27) and hence loss of ellipticity cannot be avoided under contraction if $I_{5}<1$ is sufficiently small.

We now turn our attention to $I_{5}>1$ so that $G^{\prime}>0$. As opposed to the modified form of $I_{5}$ used in [2, a necessary condition for loss of ellipticity (if the base material is strongly elliptic) is not that $G^{\prime \prime}\left(I_{5}\right)<0$ as discussed previously. It follows that under plane strain weak surfaces may be either (a) parallel to the fiber direction $(\mathbf{n} \cdot \mathbf{a}=0)$ or (b) normal to the fiber direction $(\mathbf{n}=\hat{\mathbf{a}})$. For (a) the appropriate failure mechanism is de-bonding, while in (b) it is matrix failure. On the other hand, under uniaxial tensile loading in the fiber direction, loss of ellipticity does require that convexity of the reinforcing model $G\left(I_{5}\right)$ has been lost at a prior deformation and the breakdown of ellipticity is related to matrix failure since the weak surface is normal to the fiber direction.

Consider the specialization to uniaxial deformations with $\mathbf{A}=\mathbf{i}_{1}$. From (33) it follows that $I_{5}=\lambda_{1}^{4}$, and the inequality (28) reduces to

$$
8 I_{5}^{2} G^{\prime \prime}\left(I_{5}\right) m_{1}^{2} n_{1}^{2}+G^{\prime}\left(I_{5}\right)\left[2 I_{5} n_{1}^{2}\left(1+2 m_{1}^{2}\right)+I_{3}\left(m_{1} n_{2}+m_{2} n_{1}\right)^{2}\right]>0 .
$$

We note that when $\mathbf{m} \cdot \mathbf{n}=0$, the above agrees with the corresponding expression for an incompressible material given in [6]. It is easy to show that necessary and sufficient conditions for (30) to hold are

$$
G^{\prime}\left(I_{5}\right)>0, \quad 4 I_{5} G^{\prime \prime}\left(I_{5}\right)+3 G^{\prime}\left(I_{5}\right)>0 .
$$

Furthermore, it follows that breakdown of ellipticity is related to vectors such that $\mathbf{m}=\mathbf{n}=\hat{\mathbf{a}}$. Therefore, the surface of discontinuity is normal to the fiber direction and the failure mechanism is identified as matrix failure.

We also remark that if the isotropic base material loses ellipticity, then overall ellipticity can fail either for $\mathbf{n} \cdot \mathbf{a}=0$ or $\mathbf{n}=\hat{\mathbf{a}}$. With reference to (28), it can be seen that this can occur for $G^{\prime}\left(I_{5}\right)$ and $G^{\prime \prime}\left(I_{5}\right)$ with appropriate signs.

4. Discussion and summary. This analysis has been motivated by instability phenomena in fiber-reinforced composite materials and has focused on failure prediction on the basis of loss of ellipticity of the governing differential equations under plane strain deformation for the considered elastic materials. The latter are isotropic base materials augmented by a function that accounts for the existence of fiber reinforcement (the reinforcing model). A detailed analysis of the ellipticity status of the compressible $I_{5}$ reinforcing model has been provided. In particular, it was found that loss of ellipticity (and hence fiber failure) is to be expected under fiber contraction for which $I_{5}<1$. Failure may also occur for $I_{5}>1$ under general plane strain deformation even if the reinforcing model is convex. Nevertheless, this is not the situation under uniaxial tensile loading in the fiber direction, for which loss of ellipticity does require that convexity of the reinforcing model $G\left(I_{5}\right)$ has been lost at a prior deformation. We have indicated how the breakdown of ellipticity might be related to different fiber failure mechanisms - kinking and splitting in compression and matrix failure in tension.

The compressible $I_{5}$-reinforcing model captures a failure mechanism that our previous analyses based on compressible and incompressible $I_{4}$-reinforcing models [1, 2] and a 
modified form of $I_{5}$ [2] did not capture. Now, the loss of ellipticity of compressible $I_{5}$ based reinforcing models under uniaxial tensile loading is associated with matrix failure. This allows us to conclude that matrix failure could be regarded as a compressible mechanism, in contrast to fiber kinking, which may be regarded as an isochoric mechanism.

Acknowledgement. This research was supported by the Spanish Ministerio de Ciencia y Tecnologia, PLAN NACIONAL, MAT:2002-03240.

\section{REFERENCES}

[1] J. Merodio, R.W. Ogden. Material instabilities in fiber-reinforced nonlinearly elastic solids under plane deformation. Arch. Mech. 54, 525-552 (2002). MR.1964402 (2004b:74012)

[2] J. Merodio, R.W. Ogden. Instabilities and loss of ellipticity in fiber-reinforced compressible nonlinearly elastic solids under plane deformation. Int. J. Solids Structures 40, 4707-4727 (2003). MR.1994903(2004f:74013)

[3] J. Merodio, R.W. Ogden. A note on strong ellipticity for transversely isotropic linearly elastic solids. Q. J. Mech. Appl. Math. 56, 589-591 (2003). MR2026873

[4] J. Merodio, R.W. Ogden. On tensile instabilities and ellipticity loss in fiber-reinforced incompressible nonlinearly elastic solids. Mech. Res. Comm., in press (2004).

[5] J. Merodio, R.W. Ogden. Mechanical response of fiber-reinforced incompressible nonlinearly elastic solids. Int. J. Nonlinear Mech. 40, 213-227 (2005).

[6] J. Merodio, R.W. Ogden. Tensile instabilities and ellipticity in fiber-reinforced compressible nonlinearly elastic solids. Int. J. Engng Sci., submitted (2004).

[7] C.S. Yerramalli, A.M. Waas. A nondimensional number to classify composite compressive failure. J. Appl. Mech. 71, 402-408 (2004).

[8] Ogden, R.W., 1984. Non-linear Elastic Deformations. Ellis Horwood, Chichester. MR0770388 (86h:73011)

[9] S.H. Lee, C.S. Yerramalli, A.M. Waas. Compressive splitting response of glass-fiber reinforced unidirectional composites. Composites Science and Technology 60, 2957-2966 (2000). 